\title{
Tropomyosin, the major tropical oyster Crassostrea belcheri allergen and effect of cooking on its allergenicity
}

\author{
Zailatul Hani Mohamad Yadzir ${ }^{1,2^{*}}$, Rosmilah Misnan², Faizal Bakhtiar ${ }^{1}$, Noormalin Abdullah ${ }^{1}$ \\ and Shahnaz Murad ${ }^{1}$
}

\begin{abstract}
Background: Many types of shellfish including oysters are sometime cooked before ingestion and it has been demonstrated that cooking may affect the allergenicity of food. Therefore, the aim of our present study is to identify major and minor allergens of tropical oyster (Crassostrea belcheri) and to investigate the effect of different cooking processing on the allergenicity of this oyster.
\end{abstract}

Methods: Raw, boiled, fried and roasted extracts of oyster were prepared. Protein profiles were analysed using sodium dodecyl sulphate-polyacrylamide gel electrophoresis (SDS-PAGE). Major and minor allergens and allergenicity patterns of all extracts were then determined by immunoblotting with sera from patients with positive skin prick tests (SPT) to the raw oyster extract. Mass-spectrometry was used to identify the major allergenic proteins of this oyster.

Results: SDS-PAGE of the raw extract showed 15 protein bands (20-180 kDa). In contrast, smaller numbers of protein bands were demonstrated in the boiled extract, those ranging between 40-42 and 55-150 kDa were denatured, whereas the protein profiles were altered to a similar degree by frying or roasting. The $37 \mathrm{kDa}$ proteins had the highest frequency of IgE-binding (95\%), thus identified as the major allergen of this tropical oyster. Other minor IgE-binding proteins were observed at various molecular weights. Immunoblot of raw extract yielded $11 \mathrm{lgE}$-binding proteins. The cooked extracts showed only a single lgE-binding protein at $37 \mathrm{kDa}$. Mass spectrometry analysis of the $37 \mathrm{kDa}$ major allergen identified this spot as tropomyosin.

Conclusions: Cooked extracts produce lower lgE-binding than raw extract, which suggest that thermal treatment can be used as a tool in attempting to reduce oyster allergenicity by reducing the number of IgE-reactive bands. The degree of allergenicity of this oyster was demonstrated in the order raw $>$ boiled $>$ fried $\approx$ roasted. A heat-resistent 37 $\mathrm{kDa}$ protein, corresponding to tropomyosin, was identified as the major allergen of this tropical oyster.

Keywords: Tropical oyster, Crassostrea belcheri, IgE reactivity, Tropomyosin, Major allergen, Immunoblot, Cooking processing

\section{Background}

Oysters are naturally one of the most nutritionally well balanced of foods [1]. They are low in fat, calories and cholesterol in addition to being high in protein, iron, omega 3 fatty acids, calcium, zinc and vitamin C [1].

\footnotetext{
*Correspondence: zailatul@imr.gov.my

${ }^{1}$ Allergy and Immunology Research Centre, Institute for Medical

Research, Jalan Pahang, 50588 Kuala Lumpur, Malaysia

Full list of author information is available at the end of the article
}

Unfortunately, oysters are also one of an elicitors of IgEmediated type I allergy [2-4].

It is well documented that the ingestion of oyster, skin and mucosal contact and the inhalation of aerosolized oyster proteins during cooking or in an occupational setting can cause a large variety of clinical symptoms in sensitized patients, such as urticaria, angioedema, atopic dermatitis, asthma, rhinitis, vomiting, diarrhea and anaphylaxis [2-4]. 
Currently, the only effective treatment to prevent oyster-induced allergies is a specific avoidance diet [5]. However, avoidance is often difficult due to unintentional cross-contamination or the addition of oyster as ingredients in some common sauces and condiments [5].

The identification of allergenic proteins in a particular species is an important step for the development of more accurate allergy tests and for the definition of more effective management of patients [6-8]. Until now, tropomyosin was the only well recognized allergen in oyster $[9,10]$. Tropomyosins are highly conserved proteins with a typical coiled-coil structure that are necessary for regulating muscle contraction [11-13]. The highly conserved amino acid sequence of tropomyosin is responsible for its identification as a panallergen for cross-reactivity between crustaceans, molluscs, insects and arachnids [11, 14-23].

Oyster may be consumed raw or cooked. Cooked oysters are usually subjected to some form of heat treatment including boiling, frying or roasting to enhance texture and flavours or to ensure microbiological safety. At the same time, heating can alter proteins by inducing denaturation (loss in tertiary and/or secondary interactions), formation of new intra- or inter-molecular bonds, aggregation, and/or rearrangements of disulfide bonds, as well as other conformational modifications, which can ultimately lead to changes in allergen reactivity [24]. In some cases, thermal treatment has been shown to decrease allergen reactivity [24-26], yet in other cases, thermal treatment increases allergen potency [27-29], possibly by exposing new IgE-binding sites. Therefore, understanding thermal treatment seems to be important in oyster allergenicity.

Crassostrea belcheri (tropical oyster) is a commonly consumed oyster in Malaysia. Our previous study indicated that it has provoked allergenic reactions in some individuals [30], but there has been no study about the allergenic proteins of this local oyster. The aim of this study was to identify the major allergenic proteins of the tropical oyster and to investigate the effect of different cooking methods on the allergenicity of this oyster.

\section{Methods}

\section{Preparation of tropical oyster extracts}

For the preparation of the raw oyster (Crassostrea belcheri) extract, the inner muscle tissue was homogenized in phosphate buffered saline (PBS), pH 7.2 and extracted overnight at $4{ }^{\circ} \mathrm{C}$, followed by centrifugation at 4500 and 14,000 rpm, for 30 and $15 \mathrm{~min}$, respectively. The clear supernatant was then recovered and sterilized by passing through a $0.22 \mu \mathrm{m}$ syringe filter, frozen and lyophilized. The lyophilized extracts were stored at $-20{ }^{\circ} \mathrm{C}$ until use. The boiled oyster extract was prepared by boiling muscle tissue for $10 \mathrm{~min}$ at $100{ }^{\circ} \mathrm{C}$, the fried oyster extract was carried out by frying muscle tissue with vegetable oil for $10 \mathrm{~min}$ and the roasted oyster extract was prepared by roasting at $180{ }^{\circ} \mathrm{C}$ for $10 \mathrm{~min}$ followed by homogenization according to the same protocol as above. Protein concentrations of the extracts were determined using the total protein kit (Sigma, USA), according to the manufacturer's instructions. Bovine serum albumin (BSA) was used as the protein standard.

\section{Human sera}

Sera of 20 patients with a history of oyster allergy and a positive skin prick test (SPT) to raw oyster extract were used in this study. This study was approved by the Medical Research and Ethics Committee (MREC), Ministry of Health Malaysia and informed consent was obtained from the patients or their relatives.

\section{Sodium dodecyl sulfate polyacrylamide-gel electrophoresis (SDS-PAGE), two dimensional-gel electrophoresis (2-DE) and immunoblotting}

SDS-PAGE and 2-DE were carried out as described previously $[15,16]$. The components of extracts from oyster that were separated by gel electrophoresis and recognized by IgE antibodies in sera were then analyzed by immunoblotting $[15,16]$.

\section{Mass spectrometry analysis}

The Coomassie-stained protein spots corresponding to those recognized by the above sera were manually excised and transferred to microcentrifuge tubes. These protein spots were analyzed using mass spectrometry analysis by First Base Laboratories Sdn Bhd, Malaysia. Protein samples were trypsin digested and peptides extracted according to standard techniques. Peptides were analyzed by matrix-assisted laser desorption-ionization time of flight (MALDI-TOF) mass spectrometer using a 4800 Proteomics Analyzer. Spectra were analyzed to identify proteins of interest using Mascot sequence matching software (Matrix Science) with Ludwig NR Database and taxonomy set to other metazoa.

\section{Results}

\section{Oyster protein profiles and IgE-binding proteins}

SDS-PAGE of raw oyster extract demonstrated 15 protein bands in the range of $20-180 \mathrm{kDa}$. Fewer bands were detected in the boiled extract as several protein bands between 40-42 and 55-150 kDa were sensitive to heat and thus were no longer detected in the gel. On the other hand, most of the protein bands in fried and roasted extracts were completely denatured. Therefore, they were identified as heat-sensitive proteins. In contrast, a $37 \mathrm{kDa}$ protein was very stable to heating and it can be detected in all oyster extracts even after boiling, frying or roasting (Fig. 1). 


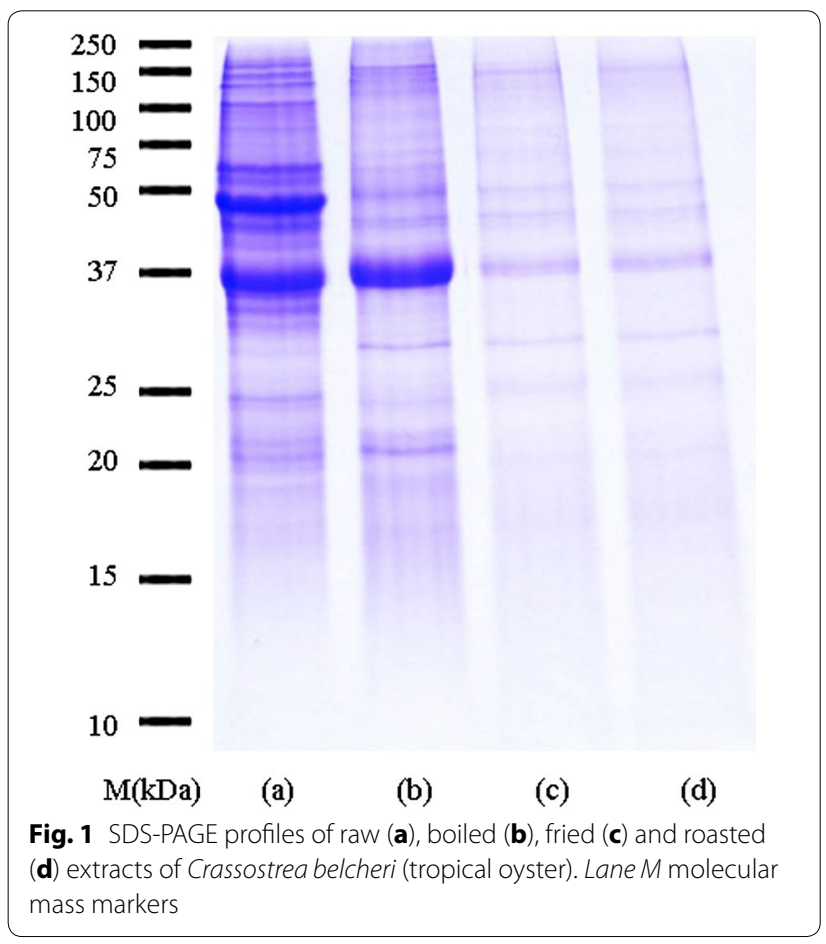

Twelve components with molecular weights ranging from about $20-180 \mathrm{kDa}$ showed IgE-binding activity in the raw oyster extract. Regarding IgE reactivity, each patient had different patterns of IgE recognition (1-12 IgE-binding bands). The component with a molecular weight of about $37 \mathrm{kDa}$ showed the highest frequency of IgE-binding (95\%) and was identified as the major allergen for this oyster (Fig. 2a). IgE-binding proteins were also detected at $5 \%(150 \mathrm{kDa}), 10 \%(33 \mathrm{kDa}), 15 \%(180$, 75, 36, 30, $20 \mathrm{kDa}), 20 \%(100 \mathrm{kDa}), 25 \%(90,65 \mathrm{kDa})$, $30 \%(25 \mathrm{kDa}), 35 \%$ (40 kDa) and $45 \%(50,42 \mathrm{kDa})$, but only as minor allergens.

On the other hand, immunoblotting of the cooked extracts detected only one IgE-binding at $37 \mathrm{kDa}$ and more staining to this band was observed in most sera tested (Fig. 2b-d). No binding was observed in the negative control and blank strip.

\section{Oyster 2-DE profiles and IgE-binding spots}

Figure 3a demonstrated the 2-dimensional gel map of proteins from the raw oyster extract. About 50 spots with molecular masses from 20 to $180 \mathrm{kDa}$ and isoelectric point $(\mathrm{pI})$ between 4 and 8 were visible with coomassie blue staining. The simple IgE-stained patterns of the oyster protein spots obtained with four sera are shown in Fig. 3b. A highly reactive protein spot with a molecular mass of $37 \mathrm{kDa}$ and $\mathrm{pI}$ of 4.57 was observed in all of the patients' serum samples tested.

\section{Allergen identification}

In the peptide mass fingerprint analysis, the excised protein spot number 1 showed the highest correlation with tropomyosin from Pacific oyster C. gigas (accession no. B7XC66) with peptide sequence coverage of $38 \%$. The corresponding sequence coverage is shown in Fig. 4.

\section{Discussion}

Cooking which can be considered a form of thermal treatment affect the allergenicity of the oyster protein. In our study, cooking processing, such as boiling, frying and roasting, produced changes on the SDS-PAGE protein profile of oyster allergens. Differences seen in these protein profiles may be attributed to temperatures at which each heat treatment was executed. Roasting and frying were performed at higher temperatures (180 and $120{ }^{\circ} \mathrm{C}$, respectively) whereas boiling was performed at relatively lower temperatures $\left(100{ }^{\circ} \mathrm{C}\right)$. Compared to the raw extracts, boiling causes a remarkable reduction in the number of protein bands between 40-42 and $55-150 \mathrm{kDa}$. Both frying and roasting showed a similar protein profile, with most of the bands clearly eliminated compared with those of the raw and boiled extracts. In contrast, a prominent heat-resistant $37 \mathrm{kDa}$ protein was detected in the SDS-PAGE even after boiling, frying or roasting. Many food allergens are resistant to denaturation, which allows them to retain the ability to elicit an immune reaction under conditions such as heating and enzymatic degradation [31].

The loss of proteins in boiled, fried and roasted extracts may be related to the effects of heat on them. Thermal treatment can potentially cause protein modifications, which include protein fragmentation, denaturation, or intra- or inter-molecular protein crosslinking [32-35]. Fragmented protein sections that are smaller than the resolution limit of the gel may travel through the acrylamide pores more quickly, causing them to ultimately be lost in the buffer. Conversely, proteins that have been modified by intermolecular crosslinking (i.e., protein aggregation) are often too large to migrate through the gel, and are not clearly detectable via SDS-PAGE, but may appear congested in the wells of the gel [32, 33, 35]. However, proteins modified via covalent modification such as intramolecular crosslinking can maintain approximately the same molecular weight, yet may often migrate through polyacrylamide gels with slight difficulty, as they are not completely linearized under reducing conditions. Thus, the protein bands corresponding to the modified proteins can have a smeared appearance in immunoblotting analyses [34]. This was apparent in Fig. 2b-d, where $37 \mathrm{kDa}$ bands were notably smeared in cooked extracts; therefore, it appears that thermal treatments may have caused oyster allergen modification via a putative 


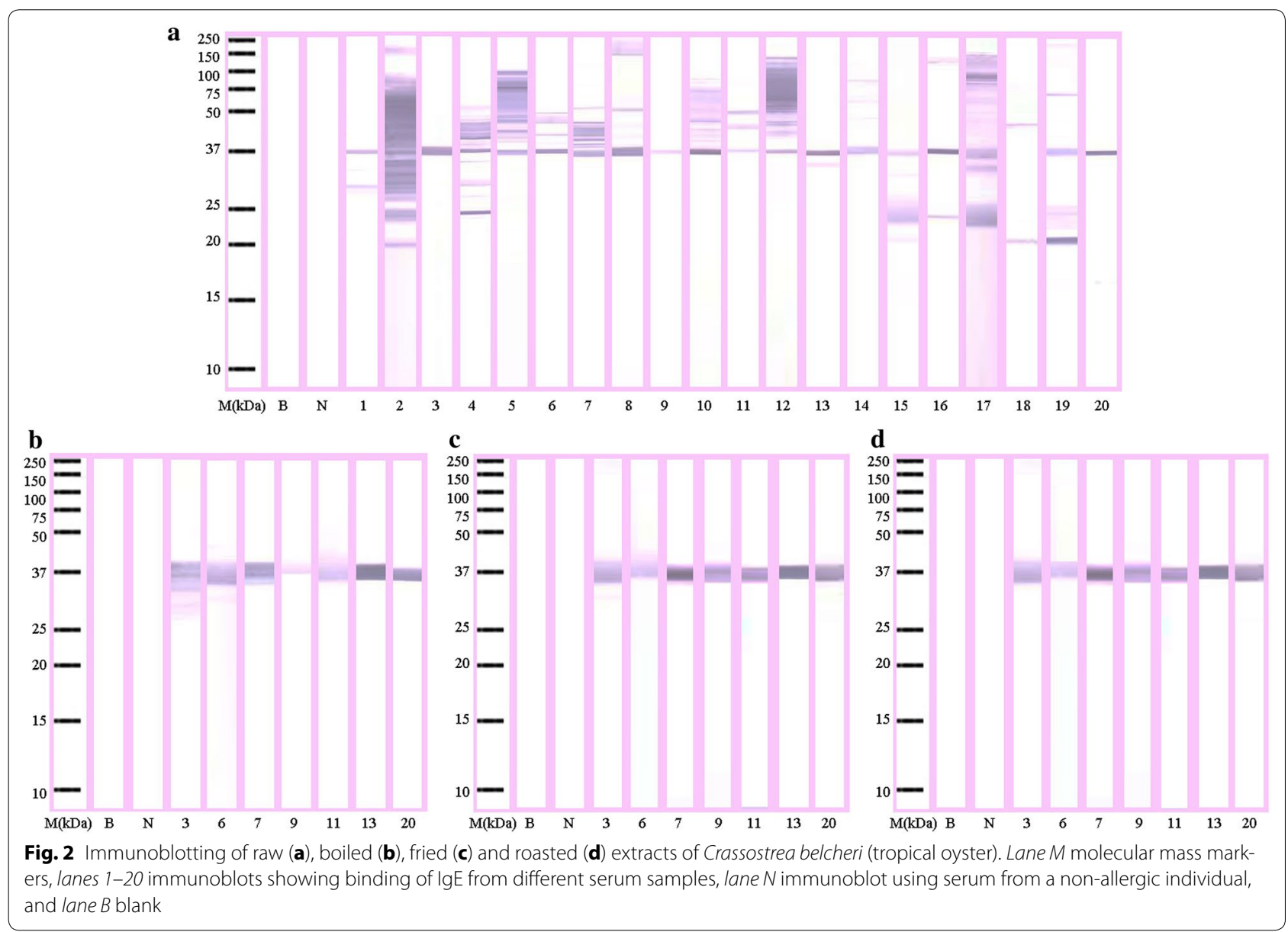

covalent modification. This speculates that the conformational modification of the $37 \mathrm{kDa}$ allergen may have changed the reactivity of the allergen by altering IgEbinding epitopes. Taheri-Kafrani et al. [33] have shown that covalent modifications, such as glycation, have been attributed to the Maillard reaction, where reducing sugars interact with proteins in a non-enzymatic browning reaction. Previous experiments using scallop tropomyosin displayed an increase in allergen potency with the progression of the Maillard reaction [36]. Kamath et al. [37] also reported that tropomyosin from oyster C. gigas has been shown to increase with heating as detected by monoclonal antibodies possibly due to the Maillard reaction. The effects of the Maillard reaction appear to be dependent on the sample and the amount and type of reducing sugars present, as well as many other variables, such as treatment temperature.

Allergens can be defined as being either major or minor. A major allergen is defined as one, recognized by IgE from more than $50 \%$ of individuals sensitive to the particular food from where the allergen originate [38]. Until now, tropomyosin was the only well recognized allergen in oyster $[9,10]$. Tropomyosin, with a molecular weight of about $34-38 \mathrm{kDa}$, is a group of highly conserved actin-binding protein present in muscle and non-muscle cells and plays a central role in muscle contraction [11-13]. Similarly, our study has also identified the $37 \mathrm{kDa}$ major allergen as tropomyosin by mass spectrometry analysis. Our findings confirmed and strengthened earlier reports on the heat stability of tropomyosin. After heat treatment, only tropomyosin exhibited the IgE-binding capacity. Tropomyosin is heat resistant and retains its IgE-binding ability even after prolonged heating $[37,39,40]$. The ability of tropomyosin to withstand heat-treatment and most known type of food processing techniques can be attributed to its exceptionally stable alpha helical coiled-coil secondary structure $[22,38]$. 


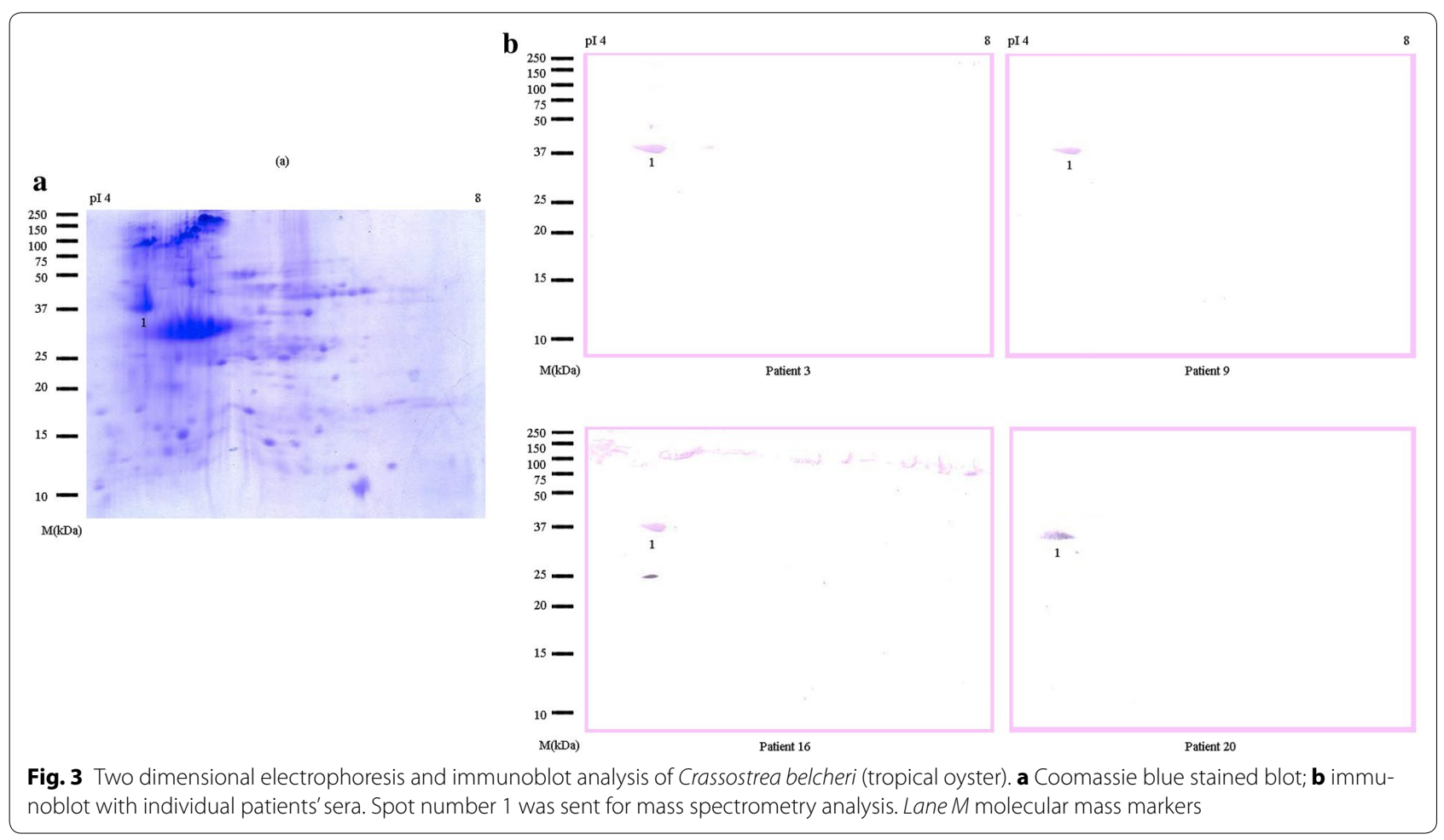

\begin{abstract}
Match to: B7XC66
Score: 1316

Number of mass values matched: 13

Sequence coverage: $38 \%$

1 MDSIKKKMIA MRMEKRNAQD RABQLEQQLR DTEEQKAKIE EDLTSLQKKH 51 SNLENEFDTV NEKYQECQTK LEEAEKTASE AEQEIQSLNR RIQLLEEDME 101 RSEERLQTAT EKLEEASKAA DESERNRKVL ENLNNASEER TDVLEKQLTE 151 AKLIAEEADK KYDEAARKLA ITEVDLERAE ARLEAAEAKV YELEEQLSVV 201 ANNIKTLQVQ NDQASQREDS YEETIRDLTQ RLKDAENRAT EAERTVSKLQ 251 KEVDRLEDEL LAEKERYKAI SDELDQTFAE LAGY
\end{abstract}

Fig. 4 Protein spot 1 peptide mass fingerprint analysis which correlates with Crassostrea gigas. Matched peptides shown in bold red

\section{Conclusions}

In conclusion, thermal treatment can be used as a tool to reduce oyster allergenicity by reducing the number of allergens. A heat-resistent $37 \mathrm{kDa}$ protein, corresponding to tropomyosin, was identified as the major allergen of this tropical oyster.

\section{Abbreviations}

SPT: Skin prick test; SDS-PAGE: sodium dodecyl sulfate polyacrylamide-gel electrophoresis; 2-DE: two dimensional-gel electrophoresis.

\section{Authors' contributions}

ZHMY: performing laboratory tests, interpretation of results and analysis, writing the paper. RM: interpretation of results and analysis, critical revision of the paper. FB: performing SPT, interpretation of results and analysis, critical revision of the paper. NA: performing SPT. SM: final approval of the paper. All authors read and approved the final manuscript.

\section{Author details}

${ }^{1}$ Allergy and Immunology Research Centre, Institute for Medical Research, Jalan Pahang, 50588 Kuala Lumpur, Malaysia. ${ }^{2}$ Department of Biology, Faculty of Science and Mathematics, Universiti Pendidikan Sultan Idris, 35900 Tanjong Malim, Perak, Malaysia.

\section{Acknowledgements}

The authors thank the Director General of Health, Malaysia for permission to publish this paper. This work was supported by a Grant UPSI 2015-0048-101-01.

\section{Competing interests}

The authors declare that they have no competing interests.

Received: 22 July 2015 Accepted: 15 October 2015

Published online: 26 October 2015

\section{References}

1. Asha KK, Anandan R, Mathew S, Lakshmanan PT. Biochemical profile of oyster Crassostrea madrasensis and its nutritional attributes. Egypt J Aquat Res. 2014;40(1):35-41.

2. Rance F, Grandmottet X, Grandjean H. Prevalence and main characteristics of school children diagnosed with food allergies in France. Clin Exp Allergy. 2005;35(2):167-72.

3. Gonzalez Galan I, Garcia Menaya JM, Jimenez Ferrera G, Gonzalez Mateos $G$. Anaphylactic shock to oysters and white fish with generalized urticaria to prawns and white fish. Allergol Immunopathol. 2002;30(5):300-3.

4. Castillo R, Delgado J, Quiralte J, Blanco C, Carillo T. Food hypersensitivity among adult patients: epidemiological and clinical aspects. Allergol Immunopathol. 1996;24(3):93-7.

5. Taylor SL. Molluscan shellfish allergy. Adv Food Nutr Res. 2008:54:139-77.

6. Ciardiello MA, Tamburrini M, Liso M, Crescenzo R, Rafaiani C, Mari A. Food allergen profiling: a big challenge. Food Res Int. 2013;54(1):1033-41. 
7. Zuidmeer-Jongejan L, Fernandez-Rivas M, Poulsen L, Neubauer A, Asturias J, Blom L, et al. FAST: towards safe and effective subcutaneous immunotherapy of persistent life-threatening food allergies. Clin Transl Allergy. 2012;2(1):5. doi:10.1186/2045-7022-2-5.

8. Woo CK, Bahna SL. Not all shellfish "allergy" is allergy! Clin Transl Allergy. 2011;1:3. doi:10.1186/2045-7022-1-3.

9. Leung PS, Chu KH. cDNA cloning and molecular identification of the major oyster allergen from the Pacific oyster Crassostrea gigas. Clin Exp Allergy. 2001;31(8):1287-94.

10. Ishikawa M, Ishida M, Shimakura K, Nagashima Y, Shiomi K. Tropomyosin, the major oyster Crassostrea gigas allergen and its IgE-binding epitopes. J Food Sci. 1998;63(1):44-7.

11. Lopata AL, Kamath S. Shellfish allergy diagnosis- gaps and needs. Curr Allergy Clin Immunol. 2012;25(2):60-6.

12. Suzuki M, Kobayashi $Y$, Hiraki Y, Nakata H, Shiomi K. Paramyosin of the disc abalone Haliotis discus discus: identification as a new allergen and crossreactivity with tropomyosin. Food Chem. 2011;124(3):921-6.

13. Thammathongchat S, Hagiwara T, Sakiyama T. Adsorption of tropomyosin from pink shrimp (Pandalus eous) on stainless steel surface. Food Control. 2010;21(9):1250-3.

14. Abramovitch JB, Kamath S, Varese N, Zubrinich C, Lopata AL, O'Hehir RE, et al. IgE reactivity of blue swimmer crab (Portunus pelagicus) Tropomyosin, Por $p 1$, and other allergens; cross-reactivity with black tiger prawn and effects of heating. PLoS One. 2013:8(6):e67487.

15. Yadzir ZH, Misnan R, Abdullah N, Bakhtiar F, Arip M, Murad S. Identification of the major allergen of Macrobrachium rosenbergii (giant freshwater prawn). Asian Pac J Trop Biomed. 2012;2(1):50-4.

16. Yadzir ZH, Misnan R, Murad S. Identification of tropomyosin as major allergen of white squid (Loligo edulis) by two-dimensional immunoblotting and mass spectrometry. Southeast Asian J Trop Med Public Health. 2012;43(1):185-91.

17. Rosmilah M, Shahnaz M, Zailatul HM, Noormalin A, Normilah I. Identification of tropomyosin and arginine kinase as major allergens of Portunus pelagicus (blue swimming crab). Trop Biomed. 2012;29(3):467-78.

18. Misnan R, Murad S, Yadzir ZH, Abdullah N. Identification of the major allergens of Charybdis feriatus (red crab) and its cross-reactivity with Portunus pelagicus (blue crab). Asian Pac J Allergy Immunol. 2012;30(4):285-93.

19. Abdel Rahman AM. Lopata AL, O'Hehir RE, Robinson JJ, Banoub JH, Helleur RJ. Characterization and de novo sequencing of snow crab tropomyosin enzymatic peptides by both electrospray ionization and matrixassisted laser desorption ionization QToF tandem mass spectrometry. J Mass Spectrom. 2010:45(4):372-81.

20. Emoto A, Ishizaki S, Shiomi K. Tropomyosins in gastropods and bivalves: identification as major allergens and amino acid sequence features. Food Chem. 2009;114(2):634-41.

21. Ayuso R, Reese G, Leong-Kee S, Plante M, Lehrer SB. Molecular basis of arthropod cross-reactivity: IgE-binding cross-reactive epitopes of shrimp, house dust mite and cockroach tropomyosins. Int Arch Allergy Immunol. 2002;129(1):38-48.

22. Reese G, Ayuso R, Lehrer SB. Tropomyosin: an invertebrate pan-allergen. Int Arch Allergy Immunol. 1999;119(4):247-58.

23. Leung PS, Chow WK, Duffey S, Kwan HS, Gershwin ME, Chu KH. IgE reactivity against a cross-reactive allergen in Crustacea and Mollusca: evidence for tropomyosin as the common allergen. J Allergy Clin Immunol. 1996:98(5 Pt 1):954-61.

24. Mondoulet L, Paty E, Drumare MF, Ah-Leung S, Scheinmann P, Willemot $\mathrm{RM}$, et al. Influence of thermal processing on the allergenicity of peanut proteins. J Agric Food Chem. 2005;53(11):4547-53.
25. Chen L, Phillips R. Effects of Twin-Screw Extrusion of Peanut Flour on In Vitro Digestion of Potentially Allergenic Peanut Proteins. J Food Protect. 2005;68(8):1712-9.

26. Beyer K, Morrow E, Li XM, Bardina L, Bannon GA, Burks AW, et al. Effects of cooking methods on peanut allergenicity. J Allergy Clin Immunol. 2001;107(6):1077-81.

27. Leszczynska J, Lacka A, Szemraj J, Lukamowicz J, Zegota H. The effect of microwave treatment on the immunoreactivity of gliadin and wheat flour. Eur Food Res Technol. 2003;217(5):387-91.

28. Simonato B, Pasini G, Giannattasio M, Peruffo ADB, De Lazzari F, Curioni A. Food allergy to wheat products: the effect of bread baking and in vitro digestion on wheat allergenic proteins. A study with bread dough, crumb, and crust. J Agr Food Chem. 2001;49(1):5668-73.

29. Pasini G, Simonato B, Giannattasio M, Peruffo ADB, Curioni A. Modifications of wheat flour proteins during in vitro digestion of bread dough, crumb, and crust: an electrophoretic and immunological study. J Agr Food Chem. 2001;49(5):2254-61.

30. Shahnaz M, Gendeh BS, Nasuruddin A. Skin test reactivity to inhalant and food allergens in patients with allergic rhinitis. J Int Med Res. 2001;5(2):69-73.

31. Ebo D, Stevens W. IgE mediated food allergy: extensive review of the literature. Acta Clin Belg. 2001;56(4):234-47.

32. Oliveira TC, Lima SL, Bressan J. Influences of different thermal processings in milk, bovine meat and frog protein structure. Nutr Hosp. 2013;28(3):896-902

33. Taheri-Kafrani A, Gaudin JC, Rabesona H, Nioi C, Aqarwal D, Drouet M, et al. Effects of heating and glycation of beta-lactoglobulin on its recognition by lgE of sera from cow milk allergy patients. J Agr Food Chem. 2009;57(11):4974-82.

34. Chatterjee U, Mondal G, Chakraborti P, Patra HK, Chatterjee BP. Changes in the allergenicity during different preparations of pomfret, hilsa, bhetki and mackerel fish as illustrated by enzyme-linked immunosorbent assay and immunoblotting. Int Arch Allergy Immunol. 2006;141(1):1-10.

35. Shriver SK, Yang WW. Thermal and nonthermal methods for food allergen control. Food Eng Rev. 2011:3:26-43.

36. Nakamura A, Watanabe K, Ojima T, Ahn DH, Saeki H. Effect of maillard reaction on allergenicity of scallop tropomyosin. J Agr Food Chem. 2009;53(19):7559-64.

37. Kamath SD. Abdel Rahman AM, Komoda T, Lopata AL. Impact of heat processing on the detection of the major shellfish allergen tropomyosin in crustaceans and molluscs using specific monoclonal antibodies. Food Chem. 2013;141(4):4031-9.

38. Bangping $W$, Zhenxing L, Lina Z, Yixuan L, Hong L. Identification and characterization of a new lgE-binding protein in mackerel (Scomber japonicus) by MALDI-TOF-MS. J Ocean Univ China. 2011;10(1):93-8.

39. Motoyama K, Suma Y, Ishizaki S, Nagashima Y, Shiomi K. Molecular cloning of tropomyosin identified as allergens in six species of crustaceans. J Agr Food Chem. 2007:55(3):985-91.

40. Leung PSC, Chen YC, Gershwin ME, Wong SH, Kwan SH, Chu KH. Identification and molecular characterization of Charybdis feriatus tropomyosin the major crab allergen. J Allergy Clin Immunol. 1998;102(5):847-52.

\section{Submit your next manuscript to BioMed Central and take full advantage of:}

- Convenient online submission

- Thorough peer review

- No space constraints or color figure charges

- Immediate publication on acceptance

- Inclusion in PubMed, CAS, Scopus and Google Scholar

- Research which is freely available for redistribution

Submit your manuscript at

www.biomedcentral.com/submit
() Biomed Central 